\title{
Elevated suicide risk among patients with urologic malignancies: highlighting at-risk populations
}

\author{
Rashid K. Sayyid ${ }^{1}$, Christopher J. D. Wallis ${ }^{2}$, Kyle Dymanus ${ }^{3}$, Usama Jazzar ${ }^{4}$, Hanan Goldberg ${ }^{5}$, \\ Stephen B. Williams ${ }^{4}$, Zachary Klaassen ${ }^{1,6}$
}

${ }^{1}$ Section of Urology, Department of Surgery, Medical College of Georgia-Augusta University, Augusta, GA, USA; ${ }^{2}$ Department of Urologic Surgery, Vanderbilt University Medical Center, Nashville, TN, USA; ${ }^{3}$ Medical College of Georgia, Augusta, GA, USA; ${ }^{4}$ Division of Urology, The University of Texas Medical Branch at Galveston, Galveston, Texas, USA; ${ }^{5}$ Department of Urology, State University of New York - Upstate, Syracuse, NY, USA; ${ }^{6}$ Georgia Cancer Center, Augusta, GA, USA

Correspondence to: Zachary Klaassen, MD, MSc. Assistant Professor Surgery/Urology, Medical College of Georgia at Augusta University, Georgia Cancer Center, Augusta, GA 30912, USA. Email: zklaassen19@gmail.com.

Provenance and Peer Review: This article was commissioned by the Editorial Office, Annals of Translational Medicine. The article did not undergo external peer review.

Comment on: Zhou H, Xian W, Zhang Y, et al. Suicide among cancer patients: adolescents and young adult (AYA) versus all-age patients. Ann Transl Med 2019;7:658.

Submitted Jan 18, 2020. Accepted for publication Feb 10, 2020.

doi: $10.21037 /$ atm.2020.02.73

View this article at: http://dx.doi.org/10.21037/atm.2020.02.73

Suicide is the tenth leading cause of death in the United States (1). It is well-established that the risk of suicide increases approximately two-fold in cancer patients, with reported rates of 31.4 and $16.7 / 100,000$ person-years in cancer patients and the general population, respectively (2). There has been improved awareness in the urologic community regarding the heightened risk of suicide amongst patients with urologic malignancies, and numerous efforts have been made to identify those specific patients at highest risk for such an event.

Zhou et al. recently reported on suicide rates of over five million patients with various solid organ malignancies, with a specific focus on the adolescent and young adult (AYA) population, using data from the Surveillance, Epidemiology, and End Results (SEER) database. The suicide mortality rates (SMR) were similar in the AYA and general populations $(0.14 \%$ vs. $0.15 \%, \mathrm{P}=0.15)$. Male sex, Caucasian race and unmarried status were associated with increased risk of suicide in both populations (3). These results are consistent with previously published reports in the urologic literature, which identified increasing age, male sex, Caucasian race, and distant disease as predictors of suicide risk in prostate, bladder, kidney, testis, and penile cancer patients (4).

Notably, the second and third highest SMRs were amongst patients with bladder $(0.37 \%)$ and prostate cancer $(0.29 \%)$, with only nasopharyngeal cancer patients $(0.38 \%)$ having a worse SMR. Furthermore, amongst all unmarried cancer patients, those with kidney and renal pelvis cancer were most likely to commit suicide in both the AYA [odds ratio $(\mathrm{OR}) 4.40,95 \% \mathrm{CI}: 1.38-16.91, \mathrm{P}=0.0017)$ and general (OR 1.97, 95\% CI: 1.52-2.56, $\mathrm{P}<0.001$ ) populations (3). These findings highlight the significantly increased risk of suicide in patients with urologic malignancies.

Patients with bladder cancer have been consistently shown to have the highest suicide rates compared to those with other urologic cancers (4). Consequently, significant efforts have been made to improve our understanding of this at-risk patient population. Using the SEER-Medicare database, Jazzar et al. evaluated the rates of post-treatment psychiatric disorders and subsequent cancer-specific and overall mortality in patients with clinical stage $\mathrm{T} 2$ through T4a bladder cancer, diagnosed between 2002 and 2011. Of 3,709 patients, 1,870 (50.4\%) were diagnosed with post-treatment psychiatric disorders. This was particularly increased in patients who underwent radical cystectomy compared to those receiving radiotherapy and/or chemotherapy [hazard ratio (HR) 1.19, 95\% CI: 1.07-1.31, $\mathrm{P}<0.01]$. Notably, those who were diagnosed with a psychiatric disorder had significantly worse cancer- 
specific (HR 2.39, 95\% CI: 2.05-2.78, $\mathrm{P}<0.01$ ) and overall survival (HR 2.80, 95\% CI: 2.47-3.17, $\mathrm{P}<0.01$ ) (5). It has been suggested that patients with psychiatric disorders are less likely to follow up with their physicians, and thus, less likely to receive appropriate, timely treatment, which then adversely affects their survival outcomes (6). Significant risk factors for suicide in the bladder cancer population mirror those in the general population (3), and included: unmarried status (HR 1.74), white race (HR 2.22), male gender (HR 6.91), presence of regional disease (HR 2.49), living in the Southeast United States (HR 2.43) and not undergoing a radical cystectomy (HR 1.42) (7).

There has also been a growing interest in the influence of pre-diagnosis psychiatric burden on outcomes in cancer patients. Using population-level data from the Canadian province of Ontario, we evaluated the impact of prediagnosis psychiatric care utilization on rates of suicide in patients diagnosed with one of the ten most common malignancies (prostate, breast, colorectal, melanoma, lung, bladder, endometrial, thyroid, kidney and oral cancer), between 1997 and 2014. The level of psychiatric care utilization within the five years prior to cancer diagnosis was used as a surrogate for psychiatric disease severity; patients were assigned a psychiatric utilization gradient (PUG) score of zero if they had no encounter with a psychiatric health care provider, one if they had an outpatient encounter, two if they had an emergency department visit related to their psychiatric disease, and three if they correspondingly required a hospital admission for psychiatric illness. Again, patients with cancer $(n=676,470)$ had a significantly increased risk of suicidal death compared to matched non-cancer controls ( $\mathrm{n}=2,152,682$; HR 1.34, 95\% CI: 1.22-1.48). This increased risk was most prominent in the first 50 months after cancer diagnosis (HR 1.60, 95\% CI: 1.42-1.81), with the rates subsequently normalizing thereafter (8). These findings emphasize the importance of providing multidisciplinary psychiatric support to these cancer patients during this initial vulnerable period where they are most at risk for suicide.

Interestingly, a cancer diagnosis in patients with a PUG score of zero or one (i.e., no encounter or outpatient encounter only) significantly increased risk of suicide, however it had no impact on suicide rates in patients with a PUG score of two or three (i.e., emergency department visit or inpatient hospitalization). These results suggest that patients with high PUG score, and thus worse psychiatric burden, are already at high risk of suicide, with a cancer diagnosis not significantly modifying this risk (8). It is those patients with a lower psychiatric burden who are most vulnerable to the effects a cancer diagnosis. Thus, efforts to target all patient populations, even those with low prediagnosis psychiatric burden, are crucial.

Using the same cohort of patients and methods to quantify pre-diagnosis psychiatric burden, it was shown that patients with higher psychiatric utilization prior to cancer diagnosis had both worse cancer-specific and allcause mortalities, with progressively worse outcomes with increasing PUG scores (6). These results are consistent with those reported by Jazzar et al. (5), and again suggest that such worse outcomes may be secondary to biologic factors and poor follow up with such patients.

Fortunately, the current data suggests that over the last few decades the risk of suicide in cancer patients is decreasing. Zhou et al. evaluated the trends of suicide mortality rates over three decades: prior to 1993, 1994 to 2003 and after 2004. The standardized mortality rates for prostate, bladder, and kidney/renal pelvis cancers all progressively decreased from $0.55 \%$ to $0.34 \%$ to $0.14 \%$, $0.46 \%$ to $0.28 \%$ to $0.16 \%$, and $0.30 \%$ to $0.16 \%$ to $0.09 \%$, respectively ( $\mathrm{P}<0.001$ for all) (3). This trend has also been demonstrated in other studies, with SEER data suggesting that diagnosis in earlier decades (1973 to 1983) was associated with a higher likelihood of suicide in patients with bladder cancer (7). This decreasing suicide rate in cancer patients is likely multifactorial in etiology. Evolving treatment strategies for cancer patients with associated improved prognoses are possibly causing less despair in these patients and, thus, less likely to result in suicide. Another potential reason may be increasing awareness of physicians regarding the vulnerability of this patient population and improved efforts to target this patient population and offer them appropriate, timely survivorship programs and support.

Nevertheless, despite these obvious improvements, further efforts are still needed to better understand why patients with psychiatric illnesses still have worse oncologic outcomes and how we can minimize the impact of cancer diagnosis on suicide risk, especially in the immediate postdiagnosis period.

\section{Acknowledgments}

Funding: None.

\section{Footnote}

Conflicts of Interest: The authors have no conflicts of interest 
to declare.

Ethical Statement: The authors are accountable for all aspects of the work in ensuring that questions related to the accuracy or integrity of any part of the work are appropriately investigated and resolved.

Open Access Statement: This is an Open Access article distributed in accordance with the Creative Commons Attribution-NonCommercial-NoDerivs 4.0 International License (CC BY-NC-ND 4.0), which permits the noncommercial replication and distribution of the article with the strict proviso that no changes or edits are made and the original work is properly cited (including links to both the formal publication through the relevant DOI and the license). See: https://creativecommons.org/licenses/by-nc-nd/4.0/.

\section{References}

1. Spicer RS, Miller TR. Suicide acts in 8 states: incidence and case fatality rates by demographics and method. Am J Public Health 2000;90:1885-91.

2. Misono S, Weiss NS, Fann JR, et al. Incidence of suicide

Cite this article as: Sayyid RK, Wallis CJD, Dymanus K, Jazzar U, Goldberg H, Williams SB, Klaassen Z. Elevated suicide risk among patients with urologic malignancies: highlighting at-risk populations. Ann Transl Med 2020;8(6):272 . doi: $10.21037 / \mathrm{atm} .2020 .02 .73$ in persons with cancer. J Clin Oncol 2008;26:4731-8.

3. Zhou H, Xian W, Zhang $\mathrm{Y}$, et al. Suicide among cancer patients: adolescents and young adult (AYA) versus all-age patients. Ann Transl Med 2019;7:658.

4. Klaassen Z, Jen RP, DiBianco JM, et al. Factors associated with suicide in patients with genitourinary malignancies. Cancer 2015;121:1864-72.

5. Jazzar U, Yong S, Klaassen Z, et al. Impact of psychiatric illness on decreased survival in elderly patients with bladder cancer in the United States. Cancer 2018;124:3127-35.

6. Klaassen Z, Wallis CJD, Goldberg H, et al. The impact of psychiatric utilisation prior to cancer diagnosis on survival of solid organ malignancies. Br J Cancer 2019;120:840-7.

7. Klaassen Z, Goldberg H, Chandrasekar T, et al. Changing Trends for Suicidal Death in Patients With Bladder Cancer: A 40+ Year Population-level Analysis. Clin Genitourin Cancer 2018;16:206-12.

8. Klaassen Z, Wallis CJD, Chandrasekar T, et al. Cancer diagnosis and risk of suicide after accounting for prediagnosis psychiatric care: A matched-cohort study of patients with incident solid-organ malignancies. Cancer 2019;125:2886-95. 